\title{
Pell-Lucas Collocation Method to Solve Second-Order Nonlinear Lane-Emden Type Pantograph Differential Equations
}

\author{
Şuayip Yüzbaşı (iD ${ }^{1 *}$, Gamze Yıldırım (iD ${ }^{1,2}$ \\ ${ }^{1}$ Akdeniz University, Faculty of Science, Department of Mathematics \\ Antalya, Türkiye \\ ${ }^{2}$ Gebze Technical University, Faculty of Basic Science, Department of Mathematics \\ Gebze, Türkiye, gamzeyildirim@akdeniz.edu.tr
}

Received: 12 December 2021

Accepted: 29 January 2022

\begin{abstract}
In this article, we present a collocation method for second-order nonlinear Lane-Emden type pantograph differential equations under intial conditions. According to the method, the solution of the problem is sought depending on the Pell-Lucas polynomials. The Pell-Lucas polynomials are written in matrix form based on the standard bases. Then, the solution form and its the derivatives are also written in matrix forms. Next, a transformation matrix is constituted for the proportion delay of the solution form. By using the matrix form of the solution, the nonlinear term in the equation is also expressed in matrix form. By using the obtained matrix forms and equally spaced collocation points, the problem is turned into an algebraic system of equations. The solution of this system gives the coefficient matrix in the solution form. In addition, the error estimation and the residual improvement technique are also presented. All presented methods are applied to three examples. The results of applications are presented in tables and graphs. In addition, the results are compared with the results of other methods in the literature.
\end{abstract}

Keywords: Collocation method, Lane-Emden differential equation, nonlinear differential equations, pantograph differential equations, Pell-Lucas polynomials.

\section{Introduction}

Many scientific phenomena are modeled with the nonlinear differential equations [10], [11]. It is not always possible to find the analytical solutions in such equations. For this reason, the numerical methods are of great importance. To date, many numerical methods are available in the literature for various types of the nonlinear differential equations [1]-[6], [9], [12]-[17], [19]-[26], [29], [35]-[51]. Yüzbaşı et. al have worked on various numerical methods by using many special polynomials until today [31]-[33], [52]-[62]. In addition, the pantograph-type differential equations have been used to characterize the problems in many fields such as physics, electronic systems,

*Correspondence: syuzbasi@akdeniz.edu.tr, suayipyuzbasi@gmail.com 2020 AMS Mathematics Subject Classification: 34A34, 65L03, 65L60, 65L80

This article is licensed under a Creative Commons Attribution 4.0 International License.

Also, it has been published considering the Research and Publication Ethics. 75 
electrodynamics, control problems, engineering, biology, population studies, infectious diseases, medicine, and economics. Many studies on the pantograph differential equations are available in the literature $[3,7,8,24,30,34,36,47]$.

On the other hand, there are studies by using the Pell-Lucas polynomials for many types of the differential equations [18], [49], [63], [64]. Since no study has yet been done by using the Pell-Lucas polynomials for the solutions of the nonlinear Lane-Emden type pantograph differential equation (LETPDE), the Pell-Lucas polynomials are used for the approximate solutions of the nonlinear second-order Lane-Emden type pantograph differential equation (LETPDE) in this study.

In this paper, we consider the second-order nonlinear Lane-Emden type pantograph differential equation (LETPDE)

$$
\gamma y^{\prime \prime}(\gamma t)+\frac{\beta}{t} y^{\prime}(\gamma t)+y^{k}(t)=g(t), \quad 0<t \leq L
$$

with the initial conditions

$$
y(0)=\lambda, \quad y^{\prime}(0)=\mu \text {. }
$$

Here, $k \in \mathbb{N}, y(t)$ is the unknown function, $g(t)$ is a continuous function $[0, L]$ and $\gamma, \beta, \lambda, \mu$ are some suitable constants. $L>0$ and $t=0$ is the single singular point of the LETPDE. Our aim is to find the approximate solution of the problem (1)-(2) in the form

$$
y(t) \approx y_{N}(t)=\sum_{n=0}^{N} a_{n} Q_{n}(t) .
$$

Here, $a_{n} \quad(n=0,1, \ldots, N)$ are the Pell Lucas coefficients, $N$ is any positive integer and $Q_{n}(t)$ are the Pell Lucas polynomials defined by

$$
Q_{n}(t)=\sum_{k=0}^{\llbracket n / 2 \rrbracket} 2^{n-2 k} \frac{n}{n-k}\left(\begin{array}{c}
n-k \\
k
\end{array}\right) t^{n-2 k} .
$$

In this study, we use two important properties of the Pell-Lucas polynomials: the recurrence relation [27], [28]

$$
Q_{n}(s)=2 s Q_{n-1}(s)+Q_{n-2}(s), \quad n \geq 2,
$$

where $Q_{0}(s)=2, Q_{1}(s)=2 s$, and the recurrence relation for the derivative [27], [28]

$$
Q_{n}^{\prime}(s)=2 s Q_{n-1}^{\prime}(s)+Q_{n-2}^{\prime}(s)+2 Q_{n-1}(s), \quad n \geq 2,
$$

where $Q_{0}^{\prime}(s)=0, Q_{1}^{\prime}(s)=2$. For more features on the Pell-Lucas polynomials, please see [27], [28].

The rest of this paper is organized as follows: The matrix form of the approximate solution and the required matrix relations are established in Section 2. The Pell-Lucas collocation method 
is constituted in Section 3. The error estimation are presented in Section 4. The applications of the method are given and the results are discussed in Section 5. In Section 6, the conclusions of the paper are given.

\section{Basic Matrix Relations}

In this section, we express the matrix forms of the problem (1)-(2) via the Pell-Lucas polynomials.

Lemma 2.1 The vector $\mathbf{Q}_{N}(t)$ can be written as

$$
\mathbf{Q}_{N}(t)=\mathbf{T}_{N}(t) \mathbf{D}_{N}
$$

where $\mathbf{T}_{N}(t)=\left[\begin{array}{lllll}1 & t & t^{2} & \cdots & t^{N}\end{array}\right]$ and if $N$ is odd

$$
\mathbf{D}_{N}^{T}=\left[\begin{array}{ccccc}
2 & 0 & 0 & \cdots & 0 \\
0 & 2^{1} \frac{1}{1}\left(\begin{array}{l}
1 \\
0
\end{array}\right) & 0 & \cdots & 0 \\
2^{0} \frac{2}{1}\left(\begin{array}{l}
1 \\
1
\end{array}\right) & 0 & 2^{2} \frac{2}{2}\left(\begin{array}{l}
2 \\
0
\end{array}\right) & \cdots & 0 \\
0 & 2^{1} \frac{3}{2}\left(\begin{array}{l}
1 \\
1
\end{array}\right) & 0 & \cdots & 0 \\
\vdots & \vdots & \vdots & \ddots & \vdots \\
0 & 2^{1} \frac{N}{\frac{N+1}{2}\left(\frac{N+1}{2}\right.}\left(\begin{array}{c}
\frac{N-1}{2} \\
0
\end{array}\right) & 0 & \cdots & 2^{N} \frac{N}{N}\left(\begin{array}{l}
N \\
0
\end{array}\right)
\end{array}\right]
$$

and if $N$ is even

$$
\mathbf{D}_{N}^{T}=\left[\begin{array}{ccccc}
2 & 0 & 0 & \cdots & 0 \\
0 & 2^{1} \frac{1}{1}\left(\begin{array}{l}
1 \\
0
\end{array}\right) & 0 & \cdots & 0 \\
2^{0} \frac{2}{1}\left(\begin{array}{l}
1 \\
1
\end{array}\right) & 0 & 2^{2} \frac{2}{2}\left(\begin{array}{l}
2 \\
0
\end{array}\right) & \cdots & 0 \\
0 & 2^{1} \frac{3}{2}\left(\begin{array}{l}
1 \\
1
\end{array}\right) & 0 & \cdots & 0 \\
\vdots & \vdots & \vdots & \ddots & \vdots \\
2^{0} \frac{N}{\frac{N}{2}(}\left(\begin{array}{c}
\frac{N}{2} \\
\frac{N}{2}
\end{array}\right) & 0 & 2^{2} \frac{N}{\frac{N+2}{2}}\left(\frac{N+2}{\frac{N-2}{2}}\right) & \cdots & 2^{N} \frac{N}{N}\left(\begin{array}{c}
N \\
0
\end{array}\right)
\end{array}\right]
$$

Proof By multiplying the vector $\mathbf{T}_{N}(t)$ by the matrix $\mathbf{D}_{N}$ from the right side, the vector $\mathbf{Q}_{N}(t)=\mathbf{T}_{N}(t) \mathbf{D}_{N}$ is obtained.

Lemma 2.2 The approximate solution based on the Pell-Lucas polynomials in (3) can be expressed in the form

$$
y(t) \approx y_{N}(t)=\mathbf{T}_{N}(t) \mathbf{D}_{N} \mathbf{A}_{N}
$$

where

$$
\mathbf{A}_{N}=\left[\begin{array}{llll}
a_{0} & a_{1} & \cdots & a_{N}
\end{array}\right]^{T}
$$

Proof By multiplying the vector $\mathbf{Q}_{N}(t)=\mathbf{T}_{N}(t) \mathbf{D}_{N}$ by the vector $\mathbf{A}_{N}$ from the right, the relation (6) is found. 
Lemma 2.3 The matrix relations for the first and second derivatives of the solution form (6) are respectively as follows

$$
\begin{aligned}
& y^{\prime}(t) \simeq y_{N}^{\prime}(t)=\boldsymbol{T}_{N}(t) \boldsymbol{B}_{N} \boldsymbol{D}_{N} \boldsymbol{A}_{N}, \\
& y^{\prime \prime}(t) \simeq y_{N}^{\prime \prime}(t)=\boldsymbol{T}_{N}(t) \boldsymbol{B}_{N}^{2} \boldsymbol{D}_{N} \boldsymbol{A}_{N},
\end{aligned}
$$

where

$$
\mathbf{B}_{N}=\left[\begin{array}{ccccc}
0 & 1 & 0 & \cdots & 0 \\
0 & 0 & 2 & \cdots & 0 \\
\vdots & \vdots & \vdots & \ddots & \vdots \\
0 & 0 & 0 & \cdots & N \\
0 & 0 & 0 & \cdots & 0
\end{array}\right]
$$

Proof When the first and second derivatives of (6) are taken, we have respectively

$$
\begin{aligned}
& y^{\prime}(t) \approx y_{N}^{\prime}(t)=\mathbf{T}_{N}^{\prime}(t) \mathbf{D}_{N} \mathbf{A}_{N} \\
& y^{\prime \prime}(t) \simeq y_{N}^{\prime \prime}(t)=\mathbf{T}_{N}^{\prime \prime}(t) \mathbf{D}_{N} \mathbf{A}_{N}
\end{aligned}
$$

Next, the first and second derivatives of $\mathbf{T}_{N}(t)$ are taken to obtain

$$
\begin{aligned}
& \mathbf{T}_{N}^{\prime}(t)=\mathbf{T}_{N}(t) \mathbf{B}_{N} \\
& \mathbf{T}_{N}^{\prime \prime}(t)=\mathbf{T}_{N}(t) \mathbf{B}_{N}^{2} .
\end{aligned}
$$

Thus, by writing the relations (9) in place of (8) respectively, we obtain the matrix relations for the first and second derivatives of the solution form (6).

Lemma 2.4 The matrix relations for the proportional delay of the first and second derivatives of the solution form (6) become respectively as follows

$$
\begin{aligned}
& y^{\prime}(\gamma t) \approx y_{N}^{\prime}(\gamma t)=\mathbf{T}_{N}(t) \mathbf{M}_{N}(\gamma) \mathbf{B}_{N} \mathbf{D}_{N} \mathbf{A}_{N} \\
& y^{\prime \prime}(\gamma t) \simeq y_{N}^{\prime \prime}(\gamma t)=\mathbf{T}_{N}(t) \mathbf{M}_{N}(\gamma) \mathbf{B}_{N}^{2} \mathbf{D}_{N} \mathbf{A}_{N}
\end{aligned}
$$

where

$$
\mathbf{M}_{N}(\gamma)=\left[\begin{array}{cccc}
(\gamma)^{0} & 0 & \cdots & 0 \\
0 & (\gamma)^{1} & \cdots & 0 \\
\vdots & \vdots & \ddots & \vdots \\
0 & 0 & \cdots & (\gamma)^{N}
\end{array}\right]
$$

Proof If $\gamma t$ is written instead of $t$ in (7), then it is achieved

$$
\begin{aligned}
& y^{\prime}(\gamma t) \approx y_{N}^{\prime}(\gamma t)=\mathbf{T}_{N}(\gamma t) \mathbf{B}_{N} \mathbf{D}_{N} \mathbf{A}_{N} \\
& y^{\prime \prime}(\gamma t) \approx y_{N}^{\prime \prime}(\gamma t)=\mathbf{T}_{N}(\gamma t) \mathbf{B}_{N}^{2} \mathbf{D}_{N} \mathbf{A}_{N}
\end{aligned}
$$

On the other hand, by multiplying the vector $\mathbf{T}_{N}(t)$ by the vector $\mathbf{M}_{N}(\gamma)$ from the right, we gain

$$
\mathbf{T}_{N}(\gamma t)=\mathbf{T}_{N}(t) \mathbf{M}_{N}(\gamma)
$$


Finally, the desired result is obtained by substituting the relation (12) in (11).

Lemma 2.5 The matrix representation of the solution (6) for the nonlinear term in (1) is expressed as follows

$$
y^{k}(t) \approx y_{N}^{k}(t)=\left(\mathbf{T}_{N}(t) \mathbf{D}_{N} \mathbf{A}_{N}\right)^{k-1}\left(\mathbf{T}_{N}(t) \mathbf{D}_{N} \mathbf{A}_{N}\right)
$$

Proof We can write $y_{N}^{k}(t)$ as

$$
y_{N}^{k}(t)=y_{N}^{k-1}(t) y_{N}(t)
$$

Then by substituting (6) in (14), the desired results is obtained.

Lemma 2.6 The matrix relations of the initial conditions (2) for the assumed solution form (6) are respectively as follows

$$
\begin{aligned}
\mathbf{U}_{N} \mathbf{A}_{N}=\lambda_{N}, & \mathbf{U}_{N}=\mathbf{T}_{N}(0) \mathbf{D}_{N}, \\
\mathbf{V}_{N} \mathbf{A}_{N}=\mu_{N}, & \mathbf{V}_{N}=\mathbf{T}_{N}(0) \mathbf{B}_{N} \mathbf{D}_{N} .
\end{aligned}
$$

Proof If 0 is written instead of $t$ in $(6)$ and $(7)$, it becomes $y(0) \simeq y_{N}(0)=\mathbf{T}_{N}(0) \mathbf{D}_{N} \mathbf{A}_{N}$ and $y^{\prime}(0) \simeq y_{N}^{\prime}(0)=\mathbf{T}_{N}(0) \mathbf{B}_{N} \mathbf{D}_{N} \mathbf{A}_{N}$. Hence, we have $\mathbf{U}_{N} \mathbf{A}_{N}=\lambda_{N}, \quad \mathbf{U}_{N}=\mathbf{T}_{N}(0) \mathbf{D}_{N}$ and $\mathbf{V}_{N} \mathbf{A}_{N}=\mu_{N}, \quad \mathbf{V}_{N}=\mathbf{T}_{N}(0) \mathbf{B}_{N} \mathbf{D}_{N}$. Consequently, the desired result is found.

Theorem 2.1 It is assumed that the solution of the problem (1)-(2) is sought in form (6). In that case, the following matrix relation is obtained

$$
\left\{\gamma \mathbf{T}_{N}(t) \mathbf{M}_{N}(\gamma) \mathbf{B}_{N}^{2} \mathbf{D}_{N}+\frac{\beta}{t} \mathbf{T}_{N}(t) \mathbf{M}_{N}(\gamma) \mathbf{B}_{N} \mathbf{D}_{N}+\left(\mathbf{T}_{N}(t) \mathbf{D}_{N} \mathbf{A}_{N}\right)^{k-1} \mathbf{T}_{N}(t) \mathbf{D}_{N}\right\} \mathbf{A}_{N}=g(t)
$$

Proof $y_{N}^{\prime \prime}(t), y_{N}^{\prime}(t)$ and $y_{N}^{k}(t)$ in (11) and (13) are substituted in (1) and thus, the proof of the theorem is completed.

\section{Method of the Solution}

The purpose of this section is to present the Pell-Lucas collocation method. For this reason, firstly, evenly spaced collocation points are defined. Next, the method is constructed by using these collocation points and the matrix relations in the previous section.

Definition 3.1 The evenly spaced collocation points are defined by

$$
t_{i}=a+\frac{b-a}{N} i, \quad i=0,1, \ldots, N
$$


Note here that: According to Theorem 3.1, these collocation points are used in (1). If the point $a$ here becomes 0 , a singularity occurs. In Theorem 3.2, the matrix forms obtained for the conditions are written instead of any two lines in the algebraic equation system obtained in Theorem 3.1. Therefore, in order to prevent the singularity that occurs when a point is 0 , instead of the row consisting of point that cause this singularity in Theorem 3.2, the matrix form formed for the first condition is written.

Theorem 3.1 Suppose that the solution of (1) is of form (6). In this instance, (1) by using the collocation points (17) is reduced to a system of nonlinear algebraic equations as follows

$$
\mathbf{W}_{N} \mathbf{A}_{N}=\mathbf{G}_{N}
$$

where

$$
\begin{aligned}
& \mathbf{W}_{N}=\left[\begin{array}{llll}
\overline{\mathbf{W}}_{N}\left(t_{0}\right) & \overline{\mathbf{W}}_{N}\left(t_{1}\right) & \cdots & \overline{\mathbf{W}}_{N}\left(t_{N}\right)
\end{array}\right]^{T}, \quad \mathbf{G}_{N}=\left[\begin{array}{llll}
g\left(t_{0}\right) & g\left(t_{1}\right) & \cdots & g\left(t_{N}\right)
\end{array}\right]^{T}, \\
& \overline{\mathbf{W}}_{N}\left(t_{i}\right)=\gamma \mathbf{T}_{N}\left(t_{i}\right) \mathbf{M}_{N}(\gamma) \mathbf{B}_{N}^{2} \mathbf{D}_{N}+\frac{\beta}{t_{i}} \mathbf{T}_{N}\left(t_{i}\right) \mathbf{M}_{N}(\gamma) \mathbf{B}_{N} \mathbf{D}_{N}+\left(\mathbf{T}_{N}\left(t_{i}\right) \mathbf{D}_{N} \mathbf{A}_{N}\right)^{k-1} \mathbf{T}_{N}\left(t_{i}\right) \mathbf{D}_{N}
\end{aligned}
$$

Proof If the collocation points (17) are written in (16), then we get

$$
\begin{aligned}
& \gamma \mathbf{T}_{N}\left(t_{i}\right) \mathbf{M}_{N}(\gamma) \mathbf{B}_{N}^{2} \mathbf{D}_{N} \mathbf{A}_{N}+\frac{\beta}{t_{i}} \mathbf{T}_{N}\left(t_{i}\right) \mathbf{M}_{N}(\gamma) \mathbf{B}_{N} \mathbf{D}_{N} \mathbf{A}_{N}+\left(\mathbf{T}_{N}\left(t_{i}\right) \mathbf{D}_{N} \mathbf{A}_{N}\right)^{k-1} \mathbf{T}_{N}\left(t_{i}\right) \mathbf{D}_{N} \mathbf{A}_{N}=g\left(t_{i}\right) \\
& \text { or } \\
& \left\{\gamma \mathbf{T}_{N}\left(t_{i}\right) \mathbf{M}_{N}(\gamma) \mathbf{B}_{N}^{2} \mathbf{D}_{N}+\frac{\beta}{t_{i}} \mathbf{T}_{N}\left(t_{i}\right) \mathbf{M}_{N}(\gamma) \mathbf{B}_{N} \mathbf{D}_{N}+\left(\mathbf{T}_{N}\left(t_{i}\right) \mathbf{D}_{N} \mathbf{A}_{N}\right)^{k-1} \mathbf{T}_{N}\left(t_{i}\right) \mathbf{D}_{N}\right\} \mathbf{A}_{N}=g\left(t_{i}\right) .
\end{aligned}
$$

We can briefly write (20) as $\mathbf{W}_{N} \mathbf{A}_{N}=\mathbf{G}_{N}$. Here,

$$
\mathbf{W}_{N}=\left[\begin{array}{llll}
\overline{\mathbf{W}}_{N}\left(t_{0}\right) & \overline{\mathbf{W}}_{N}\left(t_{1}\right) & \cdots & \overline{\mathbf{W}}_{N}\left(t_{N}\right)
\end{array}\right]^{T}
$$

and

$$
\overline{\mathbf{W}}_{N}\left(t_{i}\right)=\gamma \mathbf{T}_{N}\left(t_{i}\right) \mathbf{M}_{N}(\gamma) \mathbf{B}_{N}^{2} \mathbf{D}_{N}+\frac{\beta}{t_{i}} \mathbf{T}_{N}\left(t_{i}\right) \mathbf{M}_{N}(\gamma) \mathbf{B}_{N} \mathbf{D}_{N}+\left(\overline{\mathbf{T}}_{N}\left(t_{i}\right) \overline{\mathbf{D}}_{N} \overline{\mathbf{A}}_{N}\right)^{k-1} \mathbf{T}_{N}\left(t_{i}\right) \mathbf{D}_{N}
$$

which completes the proof of the theorem.

Theorem 3.2 It is assumed that the solution of the problem (1)-(2) is sought in type (6). Then, the problem (1)-(2) by using the collocation points (17) is reduced to a system of nonlinear algebraic equations as follows

$$
\widetilde{\mathbf{W}}_{N} \mathbf{A}_{N}=\widetilde{\mathbf{G}}_{N} .
$$


Here, $\left[\widetilde{\mathbf{W}}_{N} ; \widetilde{\mathbf{G}}_{N}\right]$ is obtained by writing the matrix forms formed for the conditions instead of any two rows in $\left[\mathbf{W}_{N} ; \mathbf{G}_{N}\right]$ in (18).

Proof A new matrix system is created by writing two equations obtained for the conditions (2) in Lemma 2.6 instead of any two rows in the system of algebraic equations in Theorem 3.1. This new system is also represented as $\widetilde{\mathbf{W}}_{N} \mathbf{A}_{N}=\widetilde{\mathbf{G}}_{N}$. The important part here is to write the row obtained for the first condition instead of the row with the singularity in the matrix $\left[\mathbf{W}_{N} ; \mathbf{G}_{N}\right]$. Thus, the proof is completed.

\section{Error Estimation}

The purpose of this section is to given an error estimation technique with the help of the residual function. In addition, by using this error estimation technique and the Pell-Lucas polynomial solution, the residual improvement technique is also presented.

Theorem 4.1 (Error Estimation) Let $y(t)$ be the exact solution and $y_{N}(t)$ be the Pell-Lucas polynomial solution with $N$-th degree of the problem (1)-(2). Then, the error problem can be obtained as follows

$$
\left\{\begin{array}{l}
\gamma e_{N}^{\prime \prime}(\gamma t)+\frac{\beta}{t} e_{N}^{\prime}(\gamma t)+\sum_{i=1}^{k}\left(\begin{array}{c}
k \\
i
\end{array}\right) e_{N}^{i}(t) y_{N}^{k-i}(t)=-R_{N}(t) \\
e_{N}(0)=0, \quad e_{N}^{\prime}(0)=0
\end{array}\right.
$$

where $e_{N}(t)=y(t)-y_{N}(t), e_{N}^{\prime}(t)=y^{\prime}(t)-y_{N}^{\prime}(t), e_{N}^{\prime}(\gamma t)=y^{\prime}(\gamma t)-y_{N}^{\prime}(\gamma t), e_{N}^{\prime \prime}(\gamma t)=y^{\prime \prime}(\gamma t)-$ $y_{N}^{\prime \prime}(\gamma t)$ and $R_{N}(t)$ is the residual function of the problem (1)-(2).

Proof Firstly, inasmuch as the Pell-Lucas polynomial solution (3) satisfies the problem (1)-(2), we can write

$$
\left\{\begin{array}{l}
R_{N}(t)=\gamma y_{N}^{\prime \prime}(\gamma t)+\frac{\beta}{t} y_{N}^{\prime}(\gamma t)+y_{N}^{k}(t)-g(t) \\
y_{N}(0)=\lambda, \quad y_{N}^{\prime}(0)=\mu
\end{array}\right.
$$

or

$$
\left\{\begin{array}{l}
R_{N}(t)+g(t)=\gamma y_{N}^{\prime \prime}(\gamma t)+\frac{\beta}{t} y_{N}^{\prime}(\gamma t)+y_{N}^{k}(t) \\
y_{N}(0)=\lambda, \quad y_{N}^{\prime}(0)=\mu
\end{array}\right.
$$

Now, let's subtract the equations in the problem (24) from equations in the problem (1)-(2), respectively, and thus we have

$$
\left\{\begin{array}{l}
\gamma e_{N}^{\prime \prime}(\gamma t)+\frac{\beta}{t} e_{N}^{\prime}(\gamma t)+y^{k}(t)-y_{N}^{k}(t)=-R_{N}(t) \\
e_{N}(0)=0, \quad e_{N}^{\prime}(0)=0
\end{array}\right.
$$

Now, since $e(t)=y(t)-y_{N}(t), y(t)$ can be expressed as $y(t)=e(t)+y_{N}(t)$. From here, we can write $y^{k}(t)=\left(e(t)+y_{N}(t)\right)^{k}$. Now, if the binomial expansion is also used in $\left(e(t)+y_{N}(t)\right)^{k}$, 
it becomes

$$
y^{k}(t)=\sum_{i=0}^{k}\left(\begin{array}{l}
k \\
i
\end{array}\right) e_{N}^{i}(t) y_{N}^{k-i}(t)
$$

Here, since this expression for $i=0$ is $y_{N}^{k}(t)$, the error problem is obtained as

$$
\left\{\begin{array}{l}
\gamma e_{N}^{\prime \prime}(\gamma t)+\frac{\beta}{t} e_{N}^{\prime}(\gamma t)+\sum_{i=1}^{k}\left(\begin{array}{c}
k \\
i
\end{array}\right) e_{N}^{i}(t) y_{N}^{k-i}(t)=-R_{N}(t) \\
e_{N}(0)=0, \quad e_{N}^{\prime}(0)=0
\end{array}\right.
$$

Hence, proof of theorem is completed.

Corollary 4.1 When the error problem (22) is solved according to the method in the Section 3, the estimated error function $e_{N, M}(t)$ is obtained.

Corollary 4.2 If the Pell-Lucas polynomial solution $y_{N}(t)$ is summed with the estimated error function $e_{N, M}(t)$, the improved approximate solution $y_{N, M}(t)$ is achieved.

Corollary 4.3 The improved error function is calculated by

$$
E_{N, M}(t)=y(t)-y_{N, M}(t)
$$

\section{Numerical Examples}

In this section, the three numerical applications are presented. These applications and the graphics are obtained with the help of Matlab program. In this section, $y(t), y_{N}(t)$ and $y_{N, M}(t)$ represent respectively the exact solution, the Pell-Lucas polynomial solution, the improved approximate solution. Also, $e_{N}(t), e_{N, M}(t)$ and $E_{N, M}(t)$ represent respectively the actual absolute error function, the estimated absolute error function and the improved absolute error function.

Example 5.1 Our first example is the nonlinear differential equation [3]

$$
\frac{1}{2} y^{\prime \prime}\left(\frac{1}{2} t\right)+\frac{3}{t} y^{\prime}\left(\frac{1}{2} t\right)+y^{2}(t)=t^{8}+2 t^{4}+3 t^{2}+1, \quad 0<t \leq 1
$$

and the initial conditions

$$
y(0)=1, \quad y^{\prime}(0)=0
$$

The exact solution of the problem (28)-(29) is $y(t)=1+t^{4}$. The Pell-Lucas polynomial solution of the problem (28)-(29) for $N=3$ is sought in the form

$$
y_{3}(t)=\sum_{n=0}^{3} a_{n} Q_{n}(t)
$$


Here, the colocation points for $N=3$ are $\left\{t_{0}=0, t_{1}=\frac{1}{3}, t_{2}=\frac{2}{3}, t_{3}=1\right\}$. Notice that the point $t_{0}=0$ creates a singularity in (28). For this reason, one of the lines created for the conditions is used instead of this line, which creates a singularity in the continuation of the method. With the help of the Theorem 3.1, the fundamental matrix equation is written as

$$
\mathbf{W}_{3} \mathbf{A}_{3}=\mathbf{G}_{3},
$$

where

$$
\begin{aligned}
& \mathbf{W}_{3}=\left[\begin{array}{llll}
\overline{\mathbf{W}}_{3}\left(t_{0}\right) & \overline{\mathbf{W}}_{3}\left(t_{1}\right) & \overline{\mathbf{W}}_{3}\left(t_{2}\right) & \overline{\mathbf{W}}_{3}\left(t_{3}\right)
\end{array}\right]^{T}, \\
& \overline{\mathbf{W}}_{3}\left(t_{i}\right)=\frac{1}{2} \mathbf{T}_{3}\left(t_{i}\right) \mathbf{M}_{3}(1 / 2) \mathbf{B}_{3}^{2} \mathbf{D}_{3}+\frac{3}{t_{i}} \mathbf{T}_{3}\left(t_{i}\right) \mathbf{M}_{3}(1 / 2) \mathbf{B}_{3} \mathbf{D}_{3}+\left(\mathbf{T}_{3}\left(t_{i}\right) \mathbf{D}_{3} \mathbf{A}_{3}\right)^{1} \mathbf{T}_{3}\left(t_{i}\right) \mathbf{D}_{3}, \\
& \mathbf{D}_{3}=\left[\begin{array}{llll}
2 & 0 & 2 & 0 \\
0 & 2 & 0 & 6 \\
0 & 0 & 4 & 0 \\
0 & 0 & 0 & 8
\end{array}\right], \quad \mathbf{G}_{3}=\left[\begin{array}{c}
1 \\
8911 / 6561 \\
18157 / 6561 \\
7
\end{array}\right], \quad \mathbf{B}_{3}=\left[\begin{array}{llll}
0 & 1 & 0 & 0 \\
0 & 0 & 2 & 0 \\
0 & 0 & 0 & 3 \\
0 & 0 & 0 & 0
\end{array}\right], \quad \mathbf{A}_{3}=\left[\begin{array}{c}
a_{0} \\
a_{1} \\
a_{2} \\
a_{3}
\end{array}\right], \\
& \mathbf{T}_{3}\left(t_{i}\right)=\left[\begin{array}{llll}
1 & t_{i} & t_{i}^{2} & t_{i}^{3}
\end{array}\right], \quad \mathbf{M}_{N}(1 / 2)=\left[\begin{array}{cccc}
1 & 0 & 0 & 0 \\
0 & 1 / 2 & 0 & 0 \\
0 & 0 & 1 / 4 & 0 \\
0 & 0 & 0 & 1 / 8
\end{array}\right]
\end{aligned}
$$

On the other hand, the matrix representations of the condition (29) are

$$
\left[\mathbf{U}_{3} ; \lambda_{3}\right]=\left[\begin{array}{cccccc}
2 & 0 & 2 & 0 & ; & 1
\end{array}\right]
$$

and

$$
\left[\mathbf{V}_{3} ; \mu_{3}\right]=\left[\begin{array}{ccccc}
0 & 2 & 0 & 6 ; & 0
\end{array}\right]
$$

Since singularity occurs in the first row of the matrix (31), the matrix $\left[\mathbf{U}_{3} ; \lambda_{3}\right]$ is written instead of this row. On the other hand, the matrix $\left[\mathbf{V}_{3} ; \mu_{3}\right]$ is written instead of the last row of the matrix (31) and thus a new algebraic matrix system called $\left[\widetilde{\mathbf{W}}_{3} ; \widetilde{\mathbf{G}}_{3}\right]$ is obtained. It should be noted that the second or third line can also be used instead of the last line here. When the nonlinear system $\left[\widetilde{\mathbf{W}}_{3} ; \widetilde{\mathbf{G}}_{3}\right]$ is solved by using the Matlab program, the Pell-Lucas coefficients matrix is calculated as follows:

$$
\mathbf{A}_{3}=\left[\begin{array}{llll}
655 / 999 & -1065 / 1838 & -450 / 2891 & 355 / 1838
\end{array}\right]^{T} .
$$

Then, the Pell-Lucas polynomial solution becomes

$$
y_{3}(t)=1.5452 e+00 t^{3}-6.2262 e-01 t^{2}-5.5511 e-17 t+1 .
$$


Table 1: Comparison of the results of the problem (28)-(29) for the Pell-Lucas collocation method and the Bernoulli collocation method [3]

\begin{tabular}{ccccc}
\hline$t_{i}$ & $\begin{array}{c}\text { The Actual Error for PM } \\
N=4\end{array}$ & $\begin{array}{c}\text { The Estimated Error for PM } \\
(N, M)=(4,6)\end{array}$ & $\begin{array}{c}\text { The Improved Error for PM } \\
(N, M)=(4,6)\end{array}$ & $\begin{array}{c}\text { The Actual Error for BCM [3] } \\
N=4\end{array}$ \\
\hline 0.1 & $1.9317 \mathrm{e}-15$ & $2.1204 \mathrm{e}-15$ & $1.8866 \mathrm{e}-16$ & $5.1070 \mathrm{e}-14$ \\
0.2 & $5.0030 \mathrm{e}-15$ & $5.2348 \mathrm{e}-15$ & $2.3175 \mathrm{e}-16$ & $1.2745 \mathrm{e}-13$ \\
0.3 & $8.0536 \mathrm{e}-15$ & $8.7198 \mathrm{e}-15$ & $6.6618 \mathrm{e}-16$ & $1.6875 \mathrm{e}-13$ \\
0.4 & $1.2899 \mathrm{e}-14$ & $1.5806 \mathrm{e}-14$ & $2.9072 \mathrm{e}-15$ & $1.8607 \mathrm{e}-13$ \\
0.5 & $2.4440 \mathrm{e}-14$ & $3.8594 \mathrm{e}-14$ & $1.4153 \mathrm{e}-14$ & $2.6268 \mathrm{e}-13$ \\
0.6 & $5.0667 \mathrm{e}-14$ & $1.1186 \mathrm{e}-13$ & $6.1191 \mathrm{e}-14$ & $5.5400 \mathrm{e}-13$ \\
0.7 & $1.0265 \mathrm{e}-13$ & $3.1564 \mathrm{e}-13$ & $2.1299 \mathrm{e}-13$ & $1.2870 \mathrm{e}-12$ \\
0.8 & $1.9456 \mathrm{e}-13$ & $8.0664 \mathrm{e}-13$ & $6.1208 \mathrm{e}-13$ & $3.7605 \mathrm{e}-12$ \\
0.9 & $3.4364 \mathrm{e}-13$ & $1.8584 \mathrm{e}-12$ & $1.5147 \mathrm{e}-12$ & $5.3457 \mathrm{e}-12$ \\
1 & $5.7022 \mathrm{e}-13$ & $3.9101 \mathrm{e}-12$ & $3.3399 \mathrm{e}-12$ & $9.4853 \mathrm{e}-12$ \\
\hline
\end{tabular}

Thus, the estimated error function for $M=4$ is written as

$$
e_{3,4}(t)=t^{4}-1.5452 e+00 t^{3}+6.2262 e-01 t^{2}+5.5511 e-17 t
$$

Figure 1 shows the exact solution and the Pell-Lucas polynomial solutions for $N=4,6,9$ of the model (28)-(29). Figure 2 displays the exact solution and the impoved Pell-Lucas polynomial solutions for $(N, M)=(4,6),(6,7),(9,10)$ of the model $(28)-(29)$. In Figure 3, the actual absolute errors functions of the model (28)-(29) for $N=4$ and $N=7$ are compared. In Figure 4, the actual absolute error function, the estimated absolute error function and the improved absolute error function for $(N, M)=(4,6)$ are compared. Table 1 indicates the actual absolute error for $N=4$, the estimated absolute error for $(N, M)=(4,6)$ and the improved absolute error for $(N, M)=(4,6)$ of the model (28)-(29). In addition, the results of the present method are compared with the results of the Bernoulli collocation method for $N=4$ in Table 1.

According to Figure 3, a better result is obtained with a larger value of $N$. According to Figure 4, the estimated absolute error function gives similar results to the actual absolute error function, and the improved absolute error function gives better results than the actual absolute error function. According to Table 1, the results of the present method give better results than the results of the Bernoulli collocation method [3].

Example 5.2 As the next example, let's consider the nonlinear differential equation

$$
\frac{1}{2} y^{\prime \prime}\left(\frac{1}{2} t\right)+\frac{1}{t} y^{\prime}\left(\frac{1}{2} t\right)+y^{2}(t)=\frac{1}{2} e^{-t / 2}-\frac{1}{t} e^{-t / 2}+e^{-2 t}
$$

and the initial conditions

$$
y(0)=1, \quad y^{\prime}(0)=-1
$$




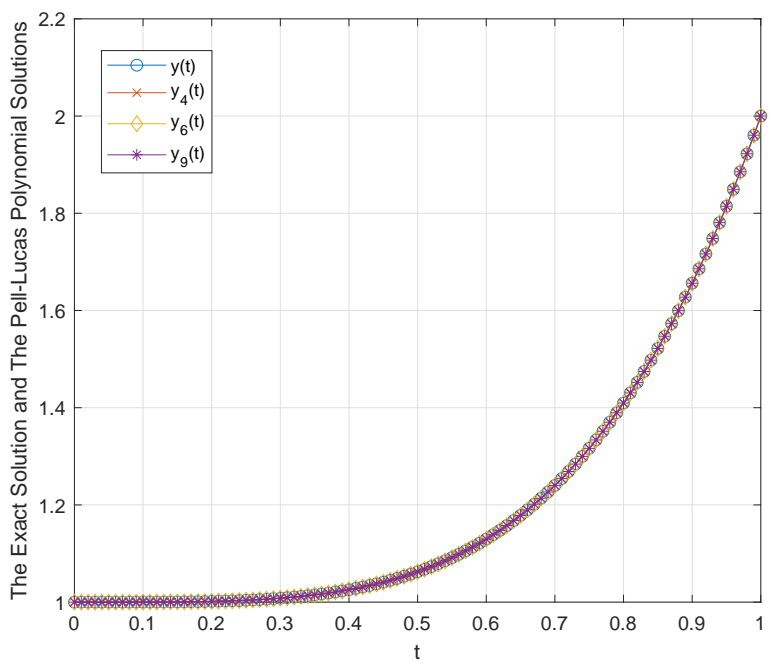

Figure 1: Comparison of the exact solution with the Pell-Lucas polynomial solutions of the problem (28)-(29) for $N=4,6,9$

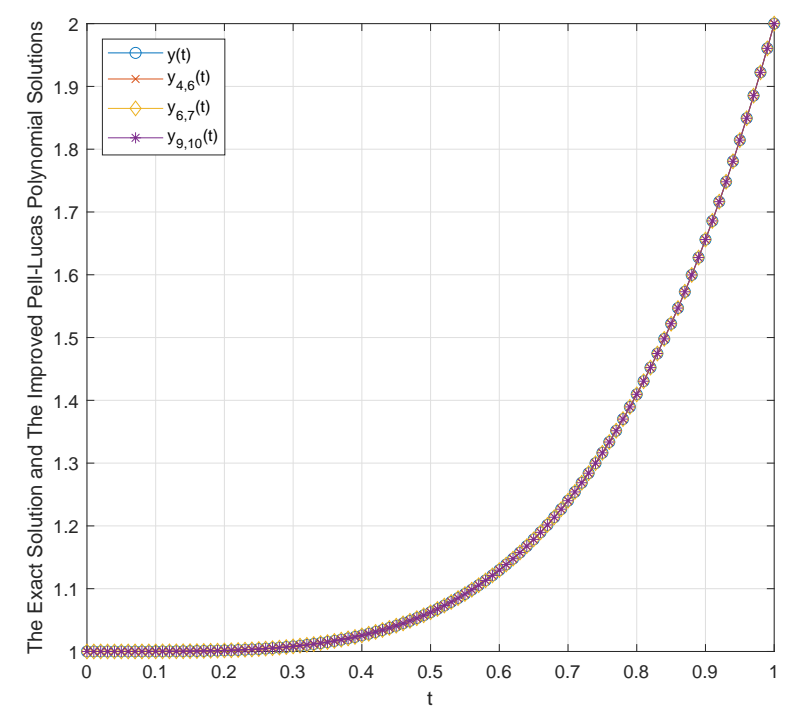

Figure 2: Comparison of the exact solution with the improved Pell-Lucas polynomial solutions of the problem $(28)-(29)$ for $(N, M)=(4,6),(N, M)=(6,7)$ and $(N, M)=(9,10)$ 


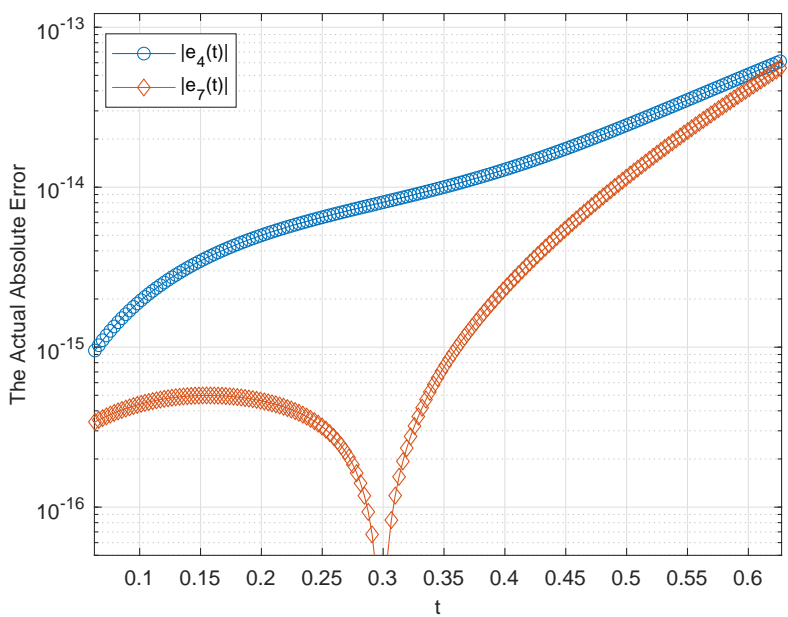

Figure 3: Comparison of the actual absolute errors of the problem (28)-(29) for $N=4$ and $N=7$

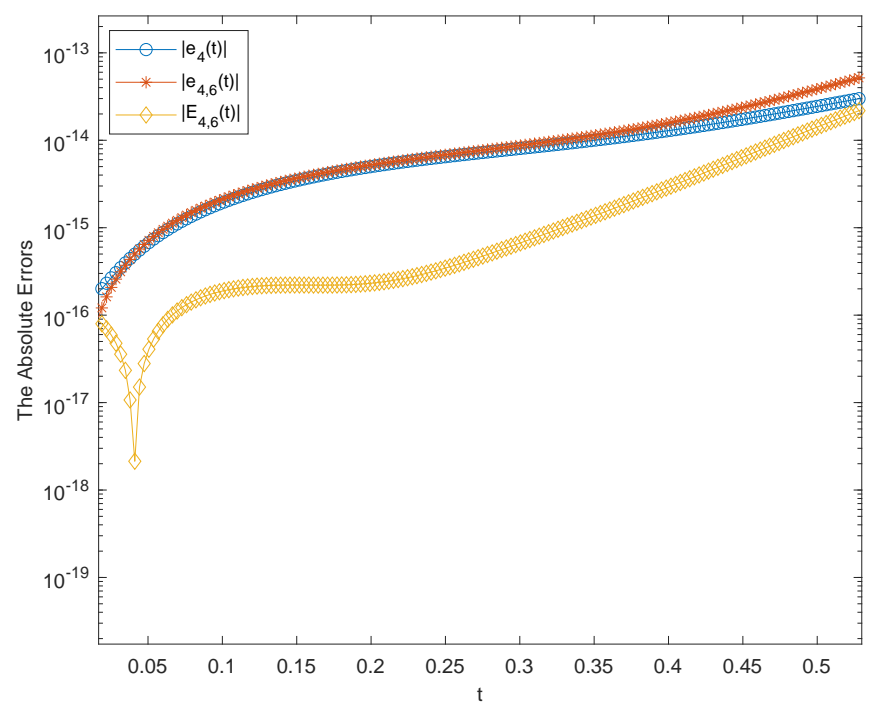

Figure 4: Comparison of the actual absolute error for $N=4$, the estimated absolute error for $(N, M)=(4,6)$ and the improved absolute error for $(N, M)=(4,6)$ of the problem (28)-(29) 
The exact solution of the problem (34)-(35) is $y(t)=e^{-t}$. The solution of the problem (34)-(35) for any $N$ is sought in the form

$$
y_{N}(t)=\sum_{n=0}^{N} a_{n} Q_{n}(t) \text {. }
$$

By using the Theorem 3.1, the fundamental matrix equation is obtained as

$$
\mathbf{W}_{N} \mathbf{A}_{N}=\mathbf{G}_{N}
$$

$$
\begin{aligned}
& \mathbf{W}_{N}=\left[\begin{array}{llll}
\overline{\mathbf{W}}_{N}\left(t_{0}\right) & \overline{\mathbf{W}}_{N}\left(t_{1}\right) & \cdots & \overline{\mathbf{W}}_{N}\left(t_{N}\right)
\end{array}\right]^{T}, \quad \mathbf{G}_{N}=\left[\begin{array}{llll}
g\left(t_{0}\right) & g\left(t_{1}\right) & \cdots & g\left(t_{N}\right)
\end{array}\right]^{T}, \\
& \overline{\mathbf{W}}_{N}\left(t_{i}\right)=\frac{1}{2} \mathbf{T}_{N}\left(t_{i}\right) \mathbf{M}_{N}(1 / 2) \mathbf{B}_{N}^{2} \mathbf{D}_{N}+\frac{1}{t_{i}} \mathbf{T}_{N}\left(t_{i}\right) \mathbf{M}_{N}(1 / 2) \mathbf{B}_{N} \mathbf{D}_{N}+\left(\mathbf{T}_{N}\left(t_{i}\right) \mathbf{D}_{N} \mathbf{A}_{N}\right)^{1} \mathbf{T}_{N}\left(t_{i}\right) \mathbf{D}_{N}
\end{aligned}
$$

The actual absolute errors, the estimated absolute errors, and the improved absolute errors of the problem (34)-(35) for various values of $N$ and $M$ are presented in Table 2. The exact solution of the problem (34)-(35) are compared with the Pell-Lucas polynomial solutions for $N=3,5,8$ in Figure 5. Also, the improved Pell-Lucas polynomial solutions are compared for $(N, M)=(3,4),(5,6),(8,9)$ in Figure 6. The actual absolute error functions of the problem (34)(35) for $N=3, N=5$ and $N=8$ are compared in Figure 7. The actual absolute error function of the problem (34)-(35) for $N=3$ is compared with the estimated absolute error function and the improved absolute error function for $(N, M)=(3,4)$ in Figure 8 .

Figure 7 shows that the errors decrease as the value of $N$ increases. From Figure 8 , it can be observed that the estimated absolute error function gives similar results to the actual absolute error function, and the improved absolute error function gives better results than the actual absolute error function. Their results can also be seen from Table 2. According to all tables and graphs, it is concluded that the method gives very successful results.

Example 5.3 The last example is the nonlinear differential equation

$$
\frac{1}{2} y^{\prime \prime}\left(\frac{1}{2} t\right)+\frac{1}{t} y^{\prime}\left(\frac{1}{2} t\right)+y^{2}(t)=-\frac{1}{2} \sin (t / 2)+\frac{1}{t} \cos (t / 2)+\sin ^{2}(t)
$$

and the initial conditions

$$
y(0)=0, \quad y^{\prime}(0)=1
$$

The exact solution of the problem (38)-(39) is $y(t)=$ sint. The solution of the problem (38)-(39) for any $N$ is sought in the form

$$
y_{N}(t)=\sum_{n=0}^{N} a_{n} Q_{n}(t) .
$$


Table 2: Absolute errors of Example 5.2 for $N=3, N=5, N=8,(N, M)=(3,4),(N, M)=(8,9)$

\begin{tabular}{|c|c|c|c|c|c|c|}
\hline \multirow[b]{2}{*}{$t_{i}$} & \multicolumn{3}{|c|}{ Actual Absolute Error } & \multicolumn{2}{|c|}{ Estimated Absolute Error } & \multirow{2}{*}{$\begin{array}{c}\text { Improved Absolute Error } \\
\qquad(N, M)=(3,4)\end{array}$} \\
\hline & $N=3$ & $N=5$ & $N=8$ & $(N, M)=(3,4)$ & $(N, M)=(8,9)$ & \\
\hline 0.1 & $1.7278 \mathrm{e}-04$ & $8.4002 \mathrm{e}-7$ & $5.2864 \mathrm{e}-11$ & $1.7663 \mathrm{e}-04$ & $4.8429 \mathrm{e}-11$ & $3.8457 \mathrm{e}-6$ \\
\hline 0.2 & $5.2756 \mathrm{e}-04$ & $1.8825 \mathrm{e}-6$ & $8.7766 \mathrm{e}-11$ & $5.4919 \mathrm{e}-04$ & $8.3974 \mathrm{e}-11$ & $2.1638 \mathrm{e}-05$ \\
\hline 0.3 & $8.8554 \mathrm{e}-04$ & $2.4972 \mathrm{e}-6$ & $1.0562 \mathrm{e}-10$ & $9.4038 \mathrm{e}-04$ & $1.0451 \mathrm{e}-10$ & $5.4837 \mathrm{e}-05$ \\
\hline 0.4 & $1.1500 \mathrm{e}-03$ & $2.8774 \mathrm{e}-6$ & $1.1801 \mathrm{e}-10$ & $1.2532 \mathrm{e}-03$ & $1.1906 \mathrm{e}-10$ & $1.0319 \mathrm{e}-04$ \\
\hline 0.5 & $1.2983 \mathrm{e}-03$ & $3.1012 \mathrm{e}-6$ & $1.2039 \mathrm{e}-10$ & $1.4709 \mathrm{e}-03$ & $1.3015 \mathrm{e}-10$ & $1.7261 \mathrm{e}-04$ \\
\hline 0.6 & $1.3751 \mathrm{e}-03$ & $2.9342 \mathrm{e}-6$ & $4.4906 \mathrm{e}-11$ & $1.6574 \mathrm{e}-03$ & $1.4307 \mathrm{e}-10$ & $2.8226 \mathrm{e}-04$ \\
\hline 0.7 & $1.4857 \mathrm{e}-03$ & $2.3052 \mathrm{e}-6$ & $1.4415 \mathrm{e}-10$ & $1.9566 \mathrm{e}-03$ & $2.8488 \mathrm{e}-10$ & $4.7093 \mathrm{e}-04$ \\
\hline 0.8 & $1.7904 \mathrm{e}-03$ & $2.3894 \mathrm{e}-6$ & $2.5977 \mathrm{e}-11$ & $2.5932 \mathrm{e}-03$ & $1.3396 \mathrm{e}-9$ & $8.0281 \mathrm{e}-04$ \\
\hline 0.9 & $2.4993 \mathrm{e}-03$ & $7.2417 \mathrm{e}-6$ & $1.5975 \mathrm{e}-9$ & $3.8721 \mathrm{e}-03$ & $5.9841 \mathrm{e}-9$ & $1.3727 \mathrm{e}-03$ \\
\hline 1 & $3.8675 \mathrm{e}-03$ & $2.5928 \mathrm{e}-05$ & $5.2919 \mathrm{e}-9$ & $6.1784 \mathrm{e}-03$ & $2.0743 \mathrm{e}-8$ & $2.3109 \mathrm{e}-03$ \\
\hline
\end{tabular}

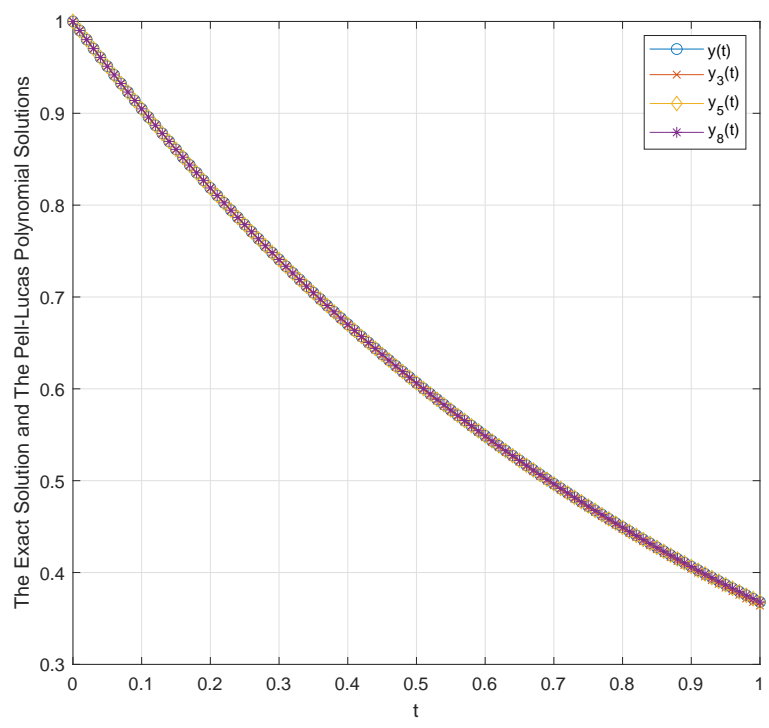

Figure 5: Comparison of the exact solution and the Pell-Lucas polynomial solutions of the problem (34)-(35) for $N=3,5,8$ 


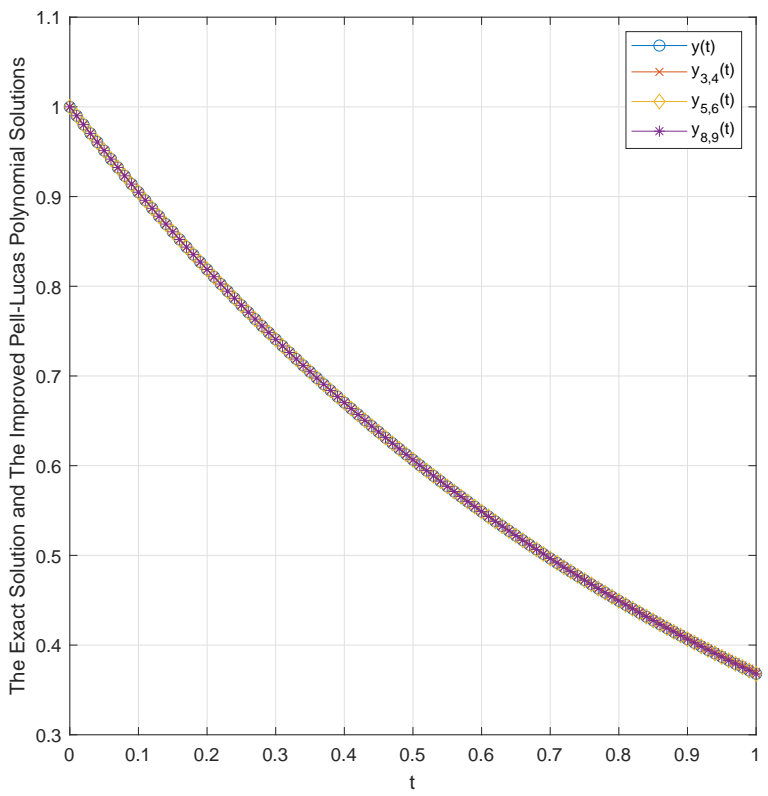

Figure 6: Comparison of the exact solution and the improved Pell-Lucas polynomial solutions of the problem $(34)-(35)$ for $(N, M)=(3,4),(N, M)=(5,6)$ and $(N, M)=(8,9)$

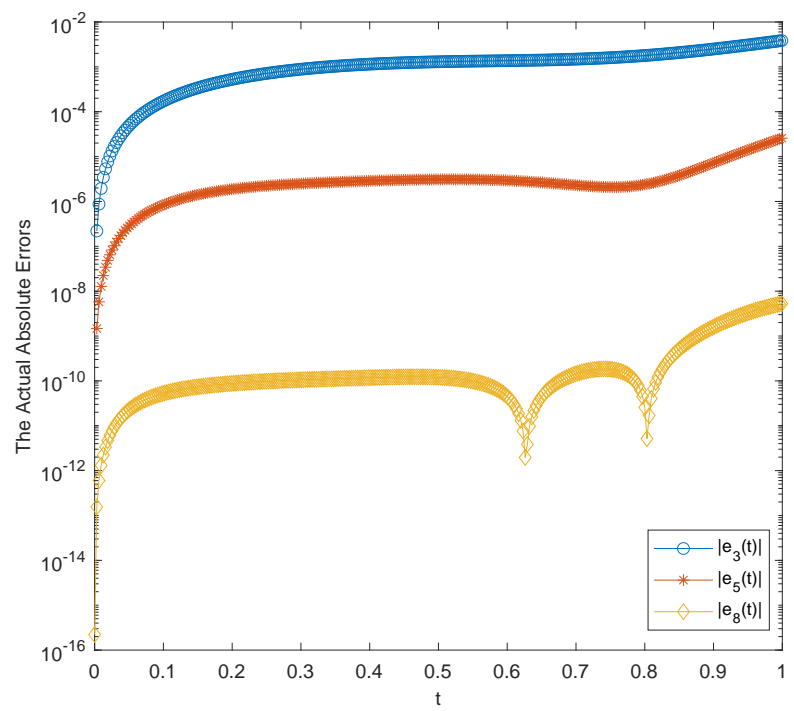

Figure 7: Comparison of the actual absolute errors of the problem (34)-(35) for $N=3,5,8$ 


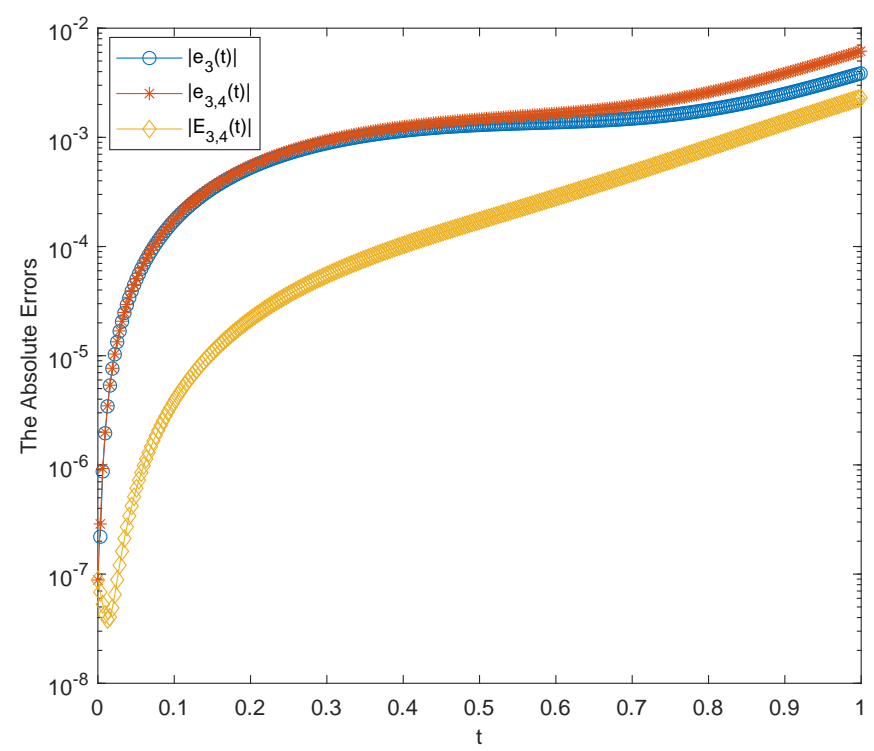

Figure 8: Comparison of the absolute errors of the problem (34)-(35) for $N=3$ and $M=4$

From the Theorem 3.1, the fundamental matrix equation becomes

$$
\mathbf{W}_{N} \mathbf{A}_{N}=\mathbf{G}_{N}
$$

where

$$
\begin{gathered}
\mathbf{W}_{N}=\left[\begin{array}{llll}
\overline{\mathbf{W}}_{N}\left(t_{0}\right) & \overline{\mathbf{W}}_{N}\left(t_{1}\right) & \cdots & \overline{\mathbf{W}}_{N}\left(t_{N}\right)
\end{array}\right]^{T}, \mathbf{G}_{N}=\left[\begin{array}{llll}
g\left(t_{0}\right) & g\left(t_{1}\right) & \cdots & g\left(t_{N}\right)
\end{array}\right]^{T}, \\
\overline{\mathbf{W}}_{N}\left(t_{i}\right)=\frac{1}{2} \mathbf{T}_{N}\left(t_{i}\right) \mathbf{M}_{N}(1 / 2) \mathbf{B}_{N}^{2} \mathbf{D}_{N}+\frac{1}{t_{i}} \mathbf{T}_{N}\left(t_{i}\right) \mathbf{M}_{N}(1 / 2) \mathbf{B}_{N} \mathbf{D}_{N}+\left(\mathbf{T}_{N}\left(t_{i}\right) \mathbf{D}_{N} \mathbf{A}_{N}\right)^{1} \mathbf{T}_{N}\left(t_{i}\right) \mathbf{D}_{N} .
\end{gathered}
$$

In Table 3, we give the actual absolute errors, the estimated absolute errors and the improved absolute errors of the problem (38)-(39) for various values of $N$ and $M$. In Figure 9 and Figure 10, we respectively compare the exact solution of the problem (38)-(39) with the approximate solutions and the improved approximate solutions. In Figure 11, we show the actual absolute error function of the problem (38)-(39) for $N=4, N=7$ and $N=9$. In Figure 12, we depict the actual absolute error function of the problem (38)-(39) for $N=3$, the estimated absolute error function and the improved absolute error function for $(N, M)=(3,4)$.

It can be observed from Figure 11 and Table 3 that a more accurate result is obtained with a larger value of $N$. The interpretation that the estimated absolute errors are very close to the actual absolute errors and that the improved absolute error function gives better results than the actual error function can be made from Figure 12 and Table 3. It is concluded from all tables and graphs that the presented method is a suitable method for the nonlinear second-order Lane-Emden type pantograph differential equation (LETPDE) (1). 
Table 3: Absolute errors for of the problem (38)-(39) $N=3, N=7, N=9,(N, M)=(3,4)$, $(N, M)=(9,10)$

\begin{tabular}{|c|c|c|c|c|c|c|}
\hline \multirow[b]{2}{*}{$t_{i}$} & \multicolumn{3}{|c|}{ The Actual Absolute Error } & \multicolumn{2}{|c|}{ The Estimated Absolute Error } & \multirow{2}{*}{$\begin{array}{l}\text { The Improved Absolute Error } \\
\qquad(N, M)=(3,4)\end{array}$} \\
\hline & $N=3$ & $N=7$ & $N=9$ & $(N, M)=(3,4)$ & $(N, M)=(9,10)$ & \\
\hline 0.1 & $7.7249 \mathrm{e}-05$ & $9.6696 \mathrm{e}-10$ & $1.0658 \mathrm{e}-12$ & $9.2330 \mathrm{e}-05$ & $8.5039 \mathrm{e}-13$ & $1.5081 \mathrm{e}-05$ \\
\hline 0.2 & $2.4963 \mathrm{e}-04$ & $1.7706 \mathrm{e}-9$ & $1.7337 \mathrm{e}-12$ & $2.8836 \mathrm{e}-04$ & $1.4459 \mathrm{e}-12$ & $3.8732 \mathrm{e}-05$ \\
\hline 0.3 & $4.3707 \mathrm{e}-04$ & $2.2067 \mathrm{e}-9$ & $2.0464 \mathrm{e}-12$ & $4.9283 \mathrm{e}-04$ & $1.7841 \mathrm{e}-12$ & $5.5762 \mathrm{e}-05$ \\
\hline 0.4 & $5.7931 \mathrm{e}-04$ & $2.4466 \mathrm{e}-9$ & $2.2737 \mathrm{e}-12$ & $6.5394 \mathrm{e}-04$ & $2.0335 \mathrm{e}-12$ & $7.4622 \mathrm{e}-05$ \\
\hline 0.5 & $6.4562 \mathrm{e}-04$ & $2.7323 \mathrm{e}-9$ & $1.9327 \mathrm{e}-12$ & $7.6354 \mathrm{e}-04$ & $2.4017 \mathrm{e}-12$ & $1.1791 \mathrm{e}-04$ \\
\hline 0.6 & $6.4414 \mathrm{e}-04$ & $3.6030 \mathrm{e}-9$ & $9.0949 \mathrm{e}-13$ & $8.5715 \mathrm{e}-04$ & $5.2066 \mathrm{e}-12$ & $2.1301 \mathrm{e}-04$ \\
\hline 0.7 & $6.3085 \mathrm{e}-04$ & $4.1655 \mathrm{e}-9$ & $4.3201 \mathrm{e}-12$ & $1.0139 \mathrm{e}-03$ & $3.1257 \mathrm{e}-11$ & $3.8309 \mathrm{e}-04$ \\
\hline 0.8 & $7.1812 \mathrm{e}-04$ & $1.9963 \mathrm{e}-10$ & $2.1487 \mathrm{e}-11$ & $1.3567 \mathrm{e}-03$ & $1.9426 \mathrm{e}-10$ & $6.3858 \mathrm{e}-04$ \\
\hline 0.9 & $1.0826 \mathrm{e}-03$ & $1.4526 \mathrm{e}-8$ & $4.8317 \mathrm{e}-11$ & $2.0519 \mathrm{e}-03$ & $9.3476 \mathrm{e}-10$ & $9.6929 \mathrm{e}-04$ \\
\hline 1 & $1.9727 \mathrm{e}-03$ & $2.3543 \mathrm{e}-8$ & $8.3901 \mathrm{e}-11$ & $3.3097 \mathrm{e}-03$ & $3.6115 \mathrm{e}-9$ & $1.3370 \mathrm{e}-03$ \\
\hline
\end{tabular}

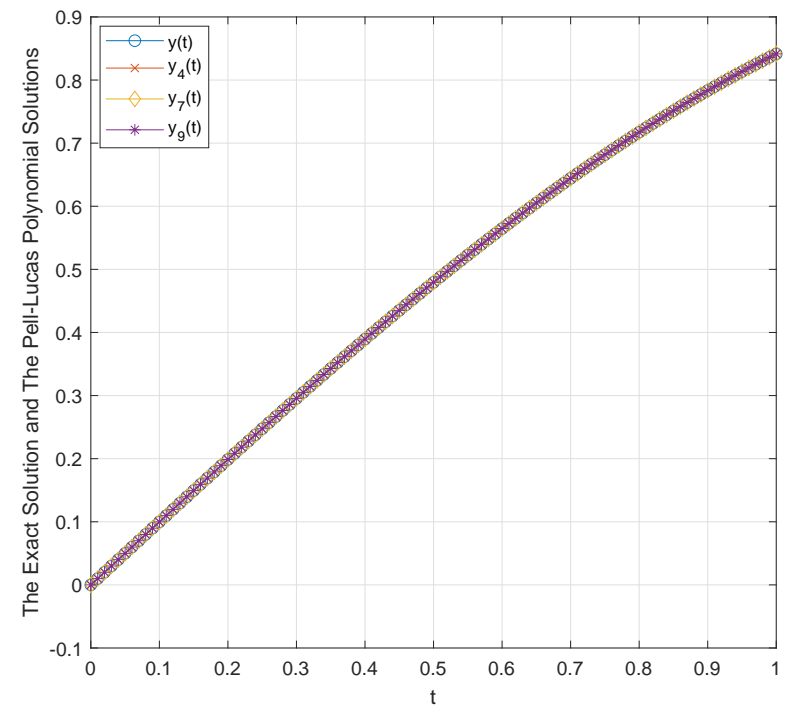

Figure 9: Comparison of the exact solution and the approximate solutions of the problem (38)-(39) for $N=4,7,9$ 


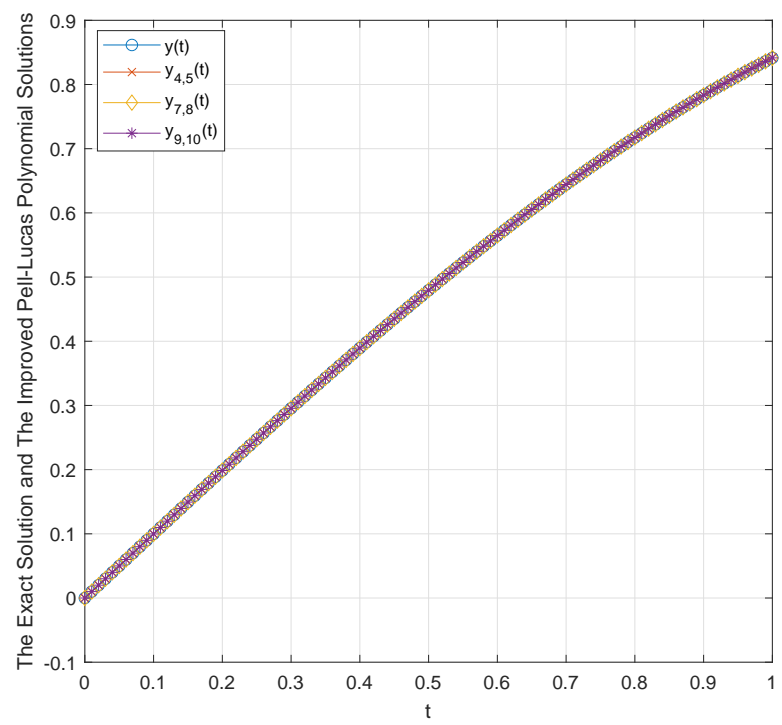

Figure 10: Comparison of the exact solution and the improved approximate solutions of the problem (38)-(39) for $(N, M)=(4,5),(N, M)=(7,8)$ and $(N, M)=(9,10)$

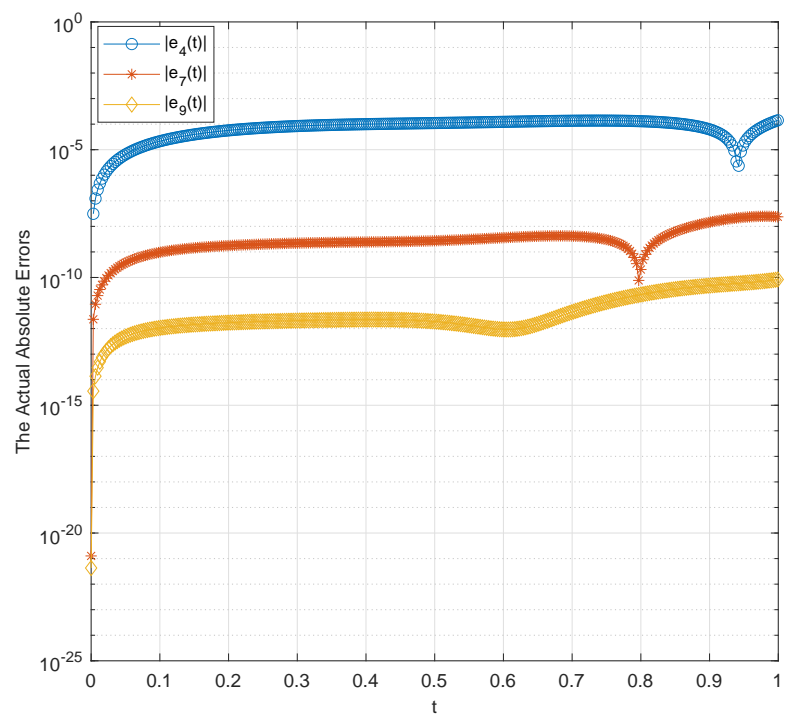

Figure 11: Comparison of the actual absolute errors of the problem (38)-(39) for $N=4,7,9$ 


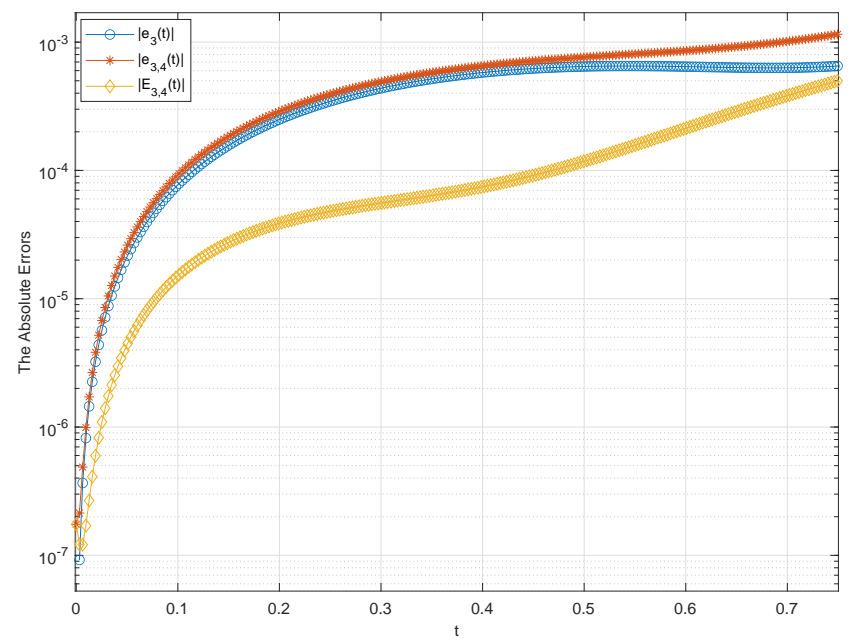

Figure 12: Comparison of the absolute errors of the problem (38)-(39) for $N=3$ and $M=4$

\section{Conclusions}

The aim of this study is to present an effective and a reliable method for a class of the nonlinear differential equations. For this purpose, the Pell-Lucas collocation method is presented in the third part of the article. In addition, in the fourth part of the article, an error estimation method is introduced by using the residual function and with the help of the third part of the article. Moreover, the residual improved technique is also presented. In the fifth section of the article, the methods presented in the previous sections are tested for three examples. These applications are made by using the Matlab program. Application results are tabulated and graphed. According to these results, it is observed that more accurate results are obtained when the value of $N$ in the method is chosen large enough. In addition, it can be said that the error estimation method is quite successful. The importance of the error estimation method is to have information about the results of the method even when the exact solution of the problem is not known. Another result of the study is that the residual improvement technique also yields appropriate results. According to all these results, it can be interpreted that the Pell-Lucas collocation method, the error estimation technique and the residual improvement method for the nonlinear second-order Lane-Emden type pantograph differential equation (LETPDE) (1) are quite effective and reliable. These presented methods can also be applied to other types of the nonlinear differential equations after the necessary adjustments are made.

\section{Declaration of Ethical Standards}

The authors declare that the materials and methods used in their study do not require ethical committee and/or legal special permission. 


\section{Authors Contributions}

Author [Şuayip Yüzbaşı]: Thought and designed the research/problem, contributed to construct the suggested method, error estimation and numerical applications (\%55).

Author [Gamze Yıldırım]: Collected the data, contributed to research method, wrote a code of the method and of solving of numerical examples and wrote the manuscript (\%45).

\section{Conflicts of Interest}

The authors declare no conflict of interest.

\section{References}

[1] Abbasbandy S., A new application of He's variational iteration method for quadratic Riccati differential equation by using Adomian's polynomials, Journal of Computational and Applied Mathematics, 207, 59-63, 2007.

[2] Abbasbandy S., Homotopy perturbation method for quadratic Riccati differential equation and comparison with Adomian's decomposition method, Applied Mathematics and Computation, 172, 485-490, 2006.

[3] Adel W., Sabir Z., Solving a new design of nonlinear second-order Lane-Emden pantograph delay differential model via Bernoulli collocation method, The European Physical Journal Plus, 135(5), 427, 2020 .

[4] Akyüz-Daşcıoğlu A., Çerdik-Yaslan H., The solution of high-order nonlinear ordinary differential equations by Chebyshev series, Applied Mathematics and Computation, 217, 5658-5666, 2011.

[5] Akyüz-Daşcioğlu A., Yaslan H.Ç., An approximation method for solution of nonlinear integral equations, Applied Mathematics and Computation, 174, 619-629, 2006.

[6] Alavizadeh S.R., Maalek Ghaini F.M., Numerical solution of higher-order linear and nonlinear ordinary differential equations with orthogonal rational Legendre functions, Journal of Mathematical Extension, 8(4), 109-130, 2014.

[7] Bahgat M.S.M., Approximate analytical solution of the linear and nonlinear multi-pantograph delay differential equations, Physica Scripta, 95(5), 055219, 2020.

[8] Bahşi M.M., Çevik M., Numerical solution of pantograph-type delay differential equations using perturbation-iteration algorithms, Journal of Applied Mathematics, 2015, Article ID 139821, 2015.

[9] Başhan A., Karakoç S.B.G., Geyikli T., Approximation of the KdVB equation by the quintic B-spline differential quadrature method, Kuwait Journal of Science, 42, 67-92, 2015.

[10] Bayin S.S., Solutions of Einstein's field equations for static fluid spheres, Physical Review D, 18, 2745-2751, 1978.

[11] Borghero F., Melis A., On Szebehely's problem for holonomic systems involving generalized potential functions, Celestial Mechanics and Dynamical Astronomy, 49, 273-284, 1990.

[12] Chen B., García-Bolós R., Jódar L., Roselló M.D., Chebyshev polynomial approximations for nonlinear differential initial value problems, Nonlinear Analysis, 63, e629-e637, 2005.

[13] Dehghan M., Abbaszadeh M., Mohebbi A., The numerical solution of nonlinear high dimensional generalized Benjamin-Bona-Mahony-Burgers equation via the meshless method of radial basis functions, Computers \& Mathematics with Applications, 68, 212-237, 2014. 
[14] Dehghan M., Manafian J., Saadatmandi A., Solving nonlinear fractional partial differential equations using the homotopy analysis method, Numerical Methods for Partial Differential Equations: An International Journal, 26, 448-479, 2010.

[15] Dehghan M., Saadatmandi A., The numerical solution of a nonlinear system of second-order boundary value problems using the sinc-collocation method, Mathematical and Computer Modelling, 46, 14341441, 2007.

[16] Dehghan M., Salehi R., The use of variational iteration method and Adomian decomposition method to solve the Eikonal equation and its application in the reconstruction problem, Communications in Numerical Methods in Engineering, 27, 524-540, 2011.

[17] Dehghan M., Shakeri F., Approximate solution of a differential equation arising in astrophysics using the variational iteration method, New Astronomy, 13, 53-59, 2008.

[18] Dönmez Demir D., Lukonde A.P., Kürkçü Ö.K., Sezer M., Pell-Lucas series approach for a class of Fredholm-type delay integro-differential equations with variable delays, Mathematical Sciences, 15, 55-64, 2021.

[19] Eftekhari A., Saadatmandi A., DE sinc-collocation method for solving a class of second-order nonlinear BVPs, Mathematics Interdisciplinary Research, 6, 11-22, 2021.

[20] El-Tawil M.A., Bahnasawi A.A., Abdel-Naby A., Solving Riccati differential equation using Adomian's decomposition method, Applied Mathematics and Computation, 157, 503-514, 2004.

[21] Eslahchi M.R., Dehghan M., Ahmadi Asl S., The general Jacobi matrix method for solving some nonlinear ordinary differential equations, Applied Mathematical Modelling, 36, 3387-3398, 2012.

[22] Genga F., Lin Y., Cui M., A piecewise variational iteration method for Riccati differential equations, Computers Mathematics with Applications, 58, 2518-2522, 2009.

[23] Geyikli T., Karakoç S.B.G., Subdomain finite element method with quartic B-splines for the modified equal width wave equation, Computational Mathematics and Mathematical Physics, 3, 410-421, 2015.

[24] Guirao J.L.G., Sabir Z., Saeed T., Design and Numerical Solutions of a Novel Third-Order Nonlinear Emden-Fowler Delay Differential Model, Mathematical Problems in Engineering, 2020, Article ID $7359242,2020$.

[25] Gümgüm S., Baykuş Savaşaneril N., Kürkçü Ö.K., Sezer M., Lucas polynomial solution of nonlinear differential equations with variable delays, Hacettepe Journal of Mathematics and Statistics, 49, 553$564,2020$.

[26] He J.H., Application of homotopy perturbation method to nonlinear wave equations, Chaos Solitons Fractals, 26, 695-700, 2005.

[27] Horadam A.F., Mahon Bro J.M., Pell and Pell-Lucas polynomials, The Fibonacci Quarterly, 23, 7-20, 1985.

[28] Horadam A.F., Swita B., Filipponi P., Integration and derivative sequences for Pell and Pell-Lucas polynomials, The Fibonacci Quarterly, 32(2), 130-135, 1994.

[29] Imani A., Aminataei A., Imani A., Collocation method via Jacobi polynomials for solving nonlinear ordinary differential equations, International Journal of Mathematics and Mathematical Sciences, 2011, Article ID 673085, 2011.

[30] Izadi M., Srivastava H.M., An efficient approximation technique applied to a non-linear Lane-Emden pantograph delay differential model, Applied Mathematics and Computation, 401, 126123, 2021.

[31] Izadi M., Yüzbaşı Ş., Baleanu D., A Taylor-Chebyshev approximation technique to solve the $1 D$ and $2 D$ nonlinear Burgers equations, Mathematical Sciences, 2021. 
[32] Izadi M., Yüzbaşı Ş., Cattani C., Approximating solutions to fractional-order Bagley-Torvik equation via generalized Bessel polynomial on large domains, Ricerche di Matematica, 2021.

[33] Izadi M., Yüzbaşı Ş., Noeiaghdam S., Approximating solutions of non-linear Troesch's problem via an efficient quasi-linearization Bessel approach, Mathematics, 9(16), 1841, 2021.

[34] Katani R., Multistep block method for linear and nonlinear pantograph type delay differential equations with neutral term, International Journal of Applied and Computational Mathematics, 3, 1347-1359, 2017.

[35] Kharrat B.N., Toma G., Differential transform method for solving initial value problems represented by strongly nonlinear ordinary differential equations, Middle-East Journal of Scientific Research, 27, 576-579, 2019.

[36] Kumar A., Methi G., An efficient numerical algorithm for solution of nonlinear delay differential equations, Journal of Physics: Conference Series, 1849, 012014, 2021.

[37] Lakestani M., Dehghan M., Numerical solution of Riccati equation using the cubic B-spline scaling functions and Chebyshev cardinalfunctions, Computer Physics Communications, 181, 957-966, 2010.

[38] Maleknejad K., Mahmoudi Y., Taylor polynomial solutions of high-order nonlinear Volterra-Fredholm integro-differential equation, Applied Mathematics and Computation, 145, 641-653, 2003.

[39] Markakis M.P., Closed-form solutions of certain Abel equations of the first kind, Applied Mathematics Letters, 22, 1401-1405, 2009.

[40] Merdan M., On the solutions of nonlinear fractional Klein-Gordon equation with modified RiemannLiouville derivative, Applied Mathematics and Computation, 242, 877-888, 2014.

[41] Mittal R.C., Jiwari R., A higher order numerical scheme for some nonlinear differential equations models in biology, International Journal of Computational Methods in Engineering Science and Mechanics, 12(3), 134-140, 2011.

[42] Noor M.A., Mohyud-Din S.T., Solution of singular and nonsingular initial and boundary value problems by modified variational iteration method, Mathematical Problems in Engineering, 2008, Article ID 917407, 2008.

[43] Noor M.A., Mohyud-Din S.T., Waheed A., Variation of parameters method for solving fifth-order boundary value problems, Applied Mathematics and Information Sciences, 2(2), 135-141, 2008.

[44] Öztürk Y., Gülsu M., The approximate solution of high-order nonlinear ordinary differential equations by improved collocation method with terms of shifted Chebyshev polynomials, International Journal of Applied and Computational Mathematics, 2, 519-531, 2016.

[45] Rawashdeh M.S., Maitama S., Solving nonlinear ordinary differential equations using the NDM, Journal of Applied Analysis and Computation, 5, 77-88, 2015.

[46] Razzaghi M., Yousefi S., Legendre wavelets method for the nonlinear Volterra-Fredholm integral equations, Mathematics and Computers in Simulation, 70, 1-8, 2005.

[47] Sabir Z., Raja M.A.Z., Le D.N., Aly A.A., A neuro-swarming intelligent heuristic for second-order nonlinear Lane-Emden multi-pantograph delay differential system, Complex \& Intelligent Systems, 2021.

[48] Sadollah A., Eskandar H., GuenYoo D., Hoon Kim J., Approximate solving of nonlinear ordinary differential equations using least square weight function and metaheuristic algorithms, Engineering Applications of Artificial Intelligence, 40, 117-132, 2015.

[49] Şahin M., Sezer M., Pell-Lucas collocation method for solving high-order functional differential equations with hybrid delays, Celal Bayar University Journal of Science, 14, 141-149, 2018. 
[50] Vanani S.K., Aminataei A., On the numerical solution of nonlinear delay differential equations, Journal of Concrete and Applicable Mathematics, 8(4), 568-576, 2010.

[51] Wazwaz A.M., El-Sayed S.M., A new modification of the Adomian decomposition method for linear and nonlinear operators, Applied Mathematics and Computation, 122, 393-404, 2001.

[52] Yüzbaşı Ş., A collocation approach to solve the Riccati-type differential equation systems, International Journal of Computer Mathematics, 89, 2180-2197, 2012.

[53] Yüzbaşı Ş., A collocation method based on Bernstein polynomials to solve nonlinear Fredholm-Volterra integro-differential equations, Applied Mathematics and Computation, 273, 142-154, 2016.

[54] Yüzbaşı Ş., An operational method for solutions of Riccati type differential equations with functional arguments, Journal of Taibah University for Science, 14, 661-669, 2020.

[55] Yüzbaşı Ş., A numerical approach for solving a class of the nonlinear Lane-Emden type equations arising in astrophysics, Mathematical Methods in the Applied Sciences, 34, 2218-2230, 2011.

[56] Yüzbaşı Ş., A numerical approximation based on the Bessel functions of first kind for solutions of Riccati type differential-difference equations, Computers \& Mathematics with Applications, 64, 1691$1705,2012$.

[57] Yüzbaşı Ş., A numerical scheme for solutions of a class of nonlinear differential equations, Journal of Taibah University for Science, 11, 1165-1181, 2017.

[58] Yüzbaşı Ş., Karaçayır M., A Galerkin-like scheme to solve Riccati equations encountered in quantum physics, Journal of Physics: Conference Series, 766, 012036, 2016.

[59] Yüzbaşı Ş., Sezer M., An exponential approach for the system of nonlinear delay integro-differential equations describing biological species living together, Neural Computing and Applications, 27, 769$779,2016$.

[60] Yüzbaşı Ş., Numerical solutions of fractional Riccati type differential equations by means of the Bernstein polynomials, Applied Mathematics and Computation, 219(11), 6328-6343, 2013.

[61] Yüzbaşı Ş., Şahin N., On the solutions of a class of nonlinear ordinary differential equations by the Bessel polynomials, Journal of Numerical Mathematics, 20, 55-79, 2012.

[62] Yüzbaşı Ş., Yıldırım G., Legendre collocation method to solve the Riccati equations with functional arguments, International Journal of Computational Methods, 17(10), 2050011, 2020.

[63] Yüzbaşı Ş., Yıldırım G., Pell-Lucas collocation method for numerical solutions of two population models and residual correction, Journal of Taibah University for Science, 14, 1262-1278, 2020.

[64] Yüzbaşı Ş., Yıldırım G., Pell-Lucas collocation method to solve high-order linear Fredholm-Volterra integro-differential equations and residual correction, Turkish Journal of Mathematics, 44(4), 10651091, 2020. 